\title{
Persistent Infection of Simian Foamy Virus Derived from the Japanese Macaque Leads to the High-Level Expression of microRNA that Resembles the miR-1 microRNA Precursor Family
}

\author{
Akira Hashimoto-Gotoh ${ }^{1}$, Koichi KitaO ${ }^{1}$, and TAKayUKi MiyaZaWA ${ }^{1,2 *}$ \\ ${ }^{1}$ Laboratory of Virus-Host Coevolution, Institute for Frontier Life and Medical Sciences, Kyoto University, Kyoto 606-8507, \\ Japan; and ${ }^{2}$ International Research Unit of Advanced Future Studies, Kyoto University, Kyoto 606-8502, Japan
}

(Received October 10, 2019—Accepted December 11, 2019_Published online January 23, 2020)

MicroRNAs (miRNAs) are a group of small non-coding RNAs that suppress the expression of target mRNAs. The seed sequence of miRNA plays a crucial role in recognizing the 3'-untranslated region of the target mRNA. Cells infected with a simian foamy virus (SFV) isolated from an African green monkey (Chlorocebus aethiops) (SFVcae) showed high expression levels of viral miRNAs encoded in the long terminal repeat of SFVcae. In the present study, we investigated the roles and expression of miRNAs derived from an SFV isolated from a Japanese macaque (Macaca fuscata) (SFVmfu) using next-generation sequencing technologies. The results obtained showed that SFVmfu also expressed viral miRNAs; however, the seed sequences of most miRNAs derived from SFVmfu differed from those reported previously from SFVcae. Cells persistently infected with SFVmfu strongly expressed an miRNA with the same seed sequence as the miR-1 microRNA precursor family. Luciferase reporter assays indicated that this miRNA down-regulates the expression of adenylyl cyclaseassociated protein 1, which is up-regulated in several solid tumors. The present results suggest that SFVmfu utilizes viral miRNAs to establish long-term co-existence with the Japanese macaque.

Key words: simian foamy virus, microRNA, miR-1 microRNA precursor family, adenylyl cyclase-associated protein 1

Simian foamy virus (SFV) is a non-pathogenetic retrovirus that belongs to the genus Simiispumavirus, sub-family Spumaviridae, family Retroviridae (Khan et al., 2018). SFV is commonly observed across the primates (Pinto-Santini et $a l ., 2017)$. Compared to other retroviruses belonging to the sub-family Orthoretroviridae, the mutation rate of foamy viruses (FVs) is extremely low $\left(1.7 \times 10^{-8}\right.$ substitutions site ${ }^{-1}$ year $^{-1}$ ) and the phylogenetic tree of SFVs from various host species is congruent with that of the hosts, which indicates that SFV undergoes co-speciation with the host primate species (Switzer et al., 2005; Liu et al., 2008). SFVs and host primates have co-existed for millions of years, which indicates that a symbiotic relationship exists between them.

MicroRNAs (miRNAs) are a group of small non-coding RNAs that are transcribed by RNA polymerase II or III (Abdelfattah et al., 2014). Processed mature miRNAs will be incorporated into a series of proteins, such as the Argonaute, to form the RNA-induced silencing complex (RISC) (Chendrimada et al., 2005). Once incorporated into RISC, one of the miRNA strands (3 prime [p] or 5p) will serve as a "guide" to recognize the complementary sequence present in the 3 '-untranslated region (3'UTR) of target mRNA, and the expression of the target gene will be repressed by RISC via deadenylation-mediated decay (Eulalio et al., 2009). The seed sequences of miRNAs (nucleotides at 2-7 or 8 bases from the 5'-terminal) are considered to be the critical component for the miRNA-induced targeting of RISC (Bartel, 2009).

Long terminal repeats (LTRs) are repetitive sequences

\footnotetext{
* Corresponding author. E-mail: takavet@infront.kyoto-u.ac.jp;
} Tel: +81-75-751-4814; Fax: +81-75-751-4814. that are present in both ends of the proviral DNA of retroviruses. LTR is composed of three regions; U3, R, and U5 (Fig. 1B): the U3 region contains the enhancer and promoter elements; the $\mathrm{R}$ region starts from the transcription start site (TSS) and contains the polyadenylation signal (Thompson et al., 2016). The lengths of the LTRs (particularly the U3 region) of FVs are longer than those of the other retroviruses. The U3 region of FVs partially overlaps with an ORF (termed ORF-2) encoding an auxiliary gene of FVs (Fig. 1B). A recent study reported that SFV LTR encodes miRNAs, which are expressed upon infection (Kincaid et al., 2014). Not only SFV, but also several retroviruses express miRNAs upon infection. These viruses include bovine leukemia virus, avian leukosis virus, human immunodeficiency virus 1, and bovine FV (Klase et al., 2007; Kincaid et al., 2012; Whisnant et al., 2014; Yao et al., 2014). miRNAs encoded in the LTRs of SFV and bovine FV include unique dumbbell-shaped pre-miRNAs that are derived from transcripts that contain two adjacent premiRNAs separated by just a few bases (Kincaid et al., 2014; Whisnant et al., 2014). The dumbbell-shaped miRNAs derived from the LTR U3 region of FVs have been shown to repress the expression of target genes (Cao et al., 2018). Therefore, the miRNAs of FVs are expected to play roles in viral replication and persistent infection; however, the functions of these miRNAs currently remain unclear.

The miRNAs derived from the LTR of SFV isolated from an African green monkey (Chlorocebus aethiops) (SFVcae) have been extensively analyzed (Kincaid et al., 2014). However, the LTR is less conserved than the other viral genes in SFVs (Galvin et al., 2013). Distinct SFVs may express miRNAs with different seed sequences that target different 
A

\begin{tabular}{cccccc}
\hline SFV-infection status & No. of total reads & Aligned 0 times & Aligned exactly 1 time & Aligned $>$ 1 time & $\begin{array}{c}\text { Overall alignment } \\
\text { of the SFVmfu genome }\end{array}$ \\
\hline Infected & 10285829 & $6991429(67.97 \%)$ & $3293348(32.02 \%)$ & $1052(0.01 \%)$ & $32.03 \%$ \\
Uninfected & 10854004 & $10853382(99.99 \%)$ & $622(0.01 \%)$ & 0 & $0.01 \%$ \\
\hline
\end{tabular}

B

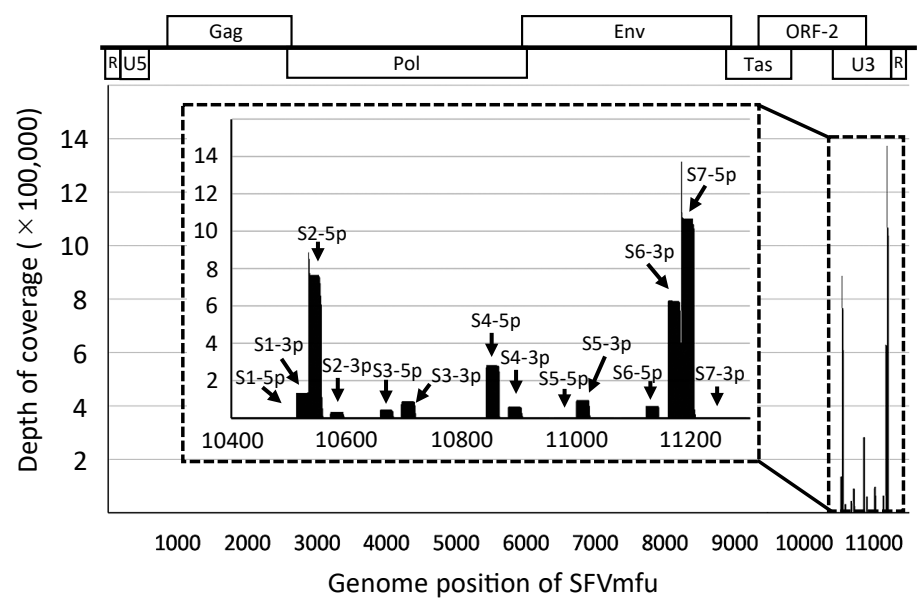

C

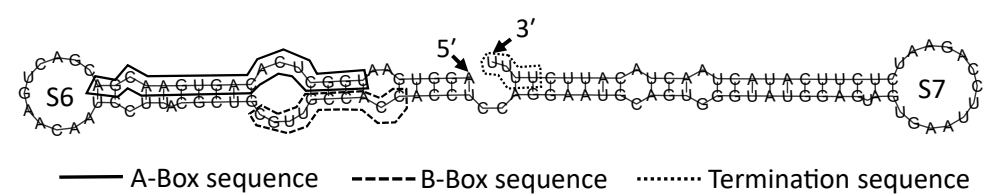

D

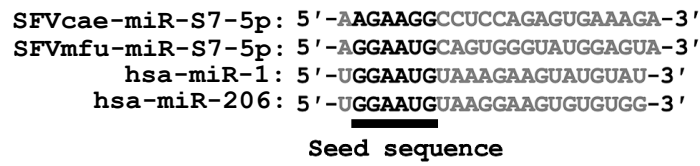

Fig. 1. Persistent infection by SFVmfu leads to the expression of miRNAs at a high level. (A) Mapping of small RNAs in TE671 and TE671/ SFVmfu(PI) cells to the genomes of SFVmfu and humans. (B) Small RNA profiling of TE671/SFVmfu(PI) cells. The diagram above the graph shows the location of SFV genes and segments of LTR used for mapping. Fourteen types of mature miRNAs are indicated with arrows. (C) Predicted secondary structure of SFVmfu-mir-S6-7 identified in this study. Circles with solid, dashed, and dotted lines indicate the predicted Abox, B-box, and termination sequences, respectively. (D) Comparison of seed sequences of miR-S7-5p of SFVmfu and SFVcae with the miR-1 miRNA precursor family (hsa-miR-1 and hsa-miR-206).

genes. We herein performed analyses of the miRNAs of SFV derived from a Japanese macaque (Macaca fuscata) (SFVmfu). This virus was previously abbreviated as SFVjm (Yoshikawa et al., 2014), which was later renamed SFVmfu (Khan et al., 2018). We revealed that these miRNAs were also expressed from the LTR of SFVmfu. Nevertheless, the seed sequences of the miRNAs derived from SFVmfu were different from those of SFVcae. Notably, an miRNA that was expressed at the highest level in SFVmfu shared the seed sequence with the miR-1 miRNA precursor family of the host. Transcriptome and immunoblot analyses and reporter assays indicated that adenylyl cyclase-associated protein 1 (CAP1) is one of the targets of the miRNA.

\section{Materials and Methods}

\section{Cells and viruses}

Human embryonic kidney (HEK) 293T (ATCC, CRL-11268) (Graham et al., 1977), TE671 (derived from human rhabdomyosarcoma) (ATCC, HTB-139) (Stratton et al., 1989), and M. dunni
(Clone III8C) cells (derived from the skin fibroblasts of Mus terricolor) (ATCC, CRL-2017) (Lander and Chattopadhyay, 1984) were cultured in Dulbecco's modified Eagle's medium (Sigma Aldrich) supplemented with $10 \%$ heat-inactivated fetal calf serum, penicillin $\left(100\right.$ units $\left.\mathrm{mL}^{-1}\right)$, and streptomycin $\left(100 \mathrm{mg} \mathrm{mL}^{-1}\right)$ (Invitrogen) at $37^{\circ} \mathrm{C}$ in a humidified atmosphere of $5 \% \mathrm{CO}_{2}$ in air. $\mathrm{An}$ SFVmfu indicator cell line (M. dunni cells encoding the $\beta$ galactosidase gene driven by the SFVmfu LTR), termed MD(SFVmfu-lacZ) cells, was newly established using the same approach as that described previously (Lambert et al., 2016). $\mathrm{MD}$ (SFVmfu-lacZ) cells were cultured in the same medium as that described above, except that puromycin $\left(5 \mu \mathrm{g} \mathrm{mL}^{-1}\right)$ was added. To prepare stock viruses of the SFVmfu strain WK1, M. dunni cells were transfected with an infectious molecular clone (named pJM356) (Yoshikawa et al., 2014) of the strain using Lipofectamine 2000 (Thermo Fisher Scientific) according to the manufacturer's instructions. Transfected cells were further cultured until syncytial foci became prominent. Cells were then freeze-thawed three times and sonicated to release intracellular virus particles. Cell debris was removed by centrifugation at 3,000 rpm for $10 \mathrm{~min}$, and viruses were collected as stock viruses by filtrating with 450-nm membrane filters (GVS). Stock viruses were stored at $-80^{\circ} \mathrm{C}$ until used. 


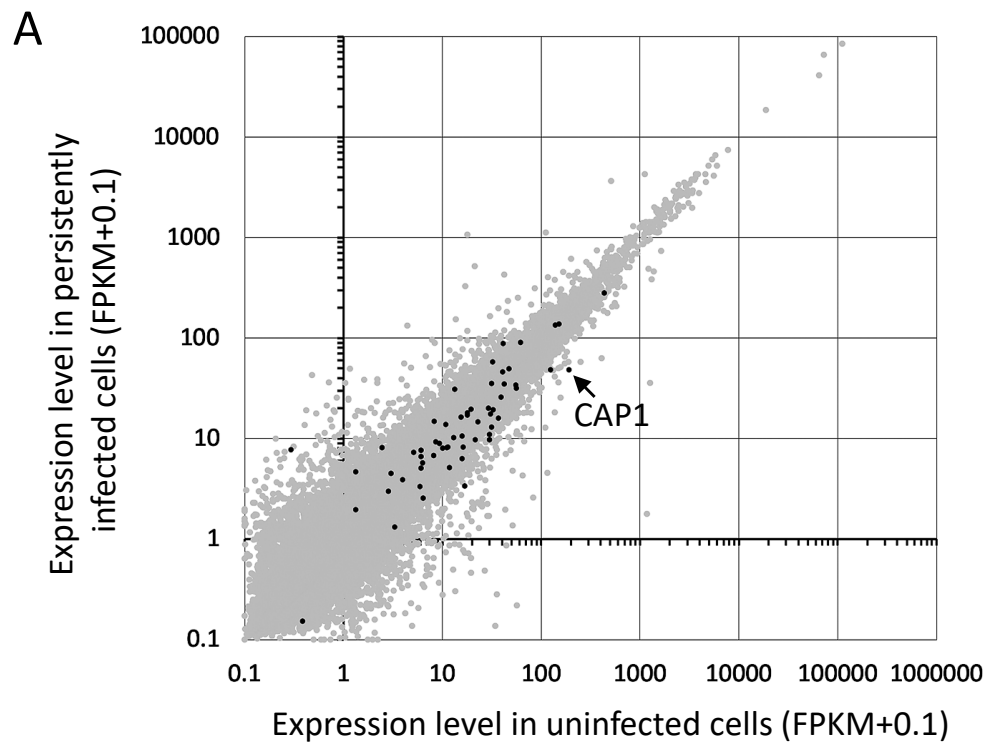

B

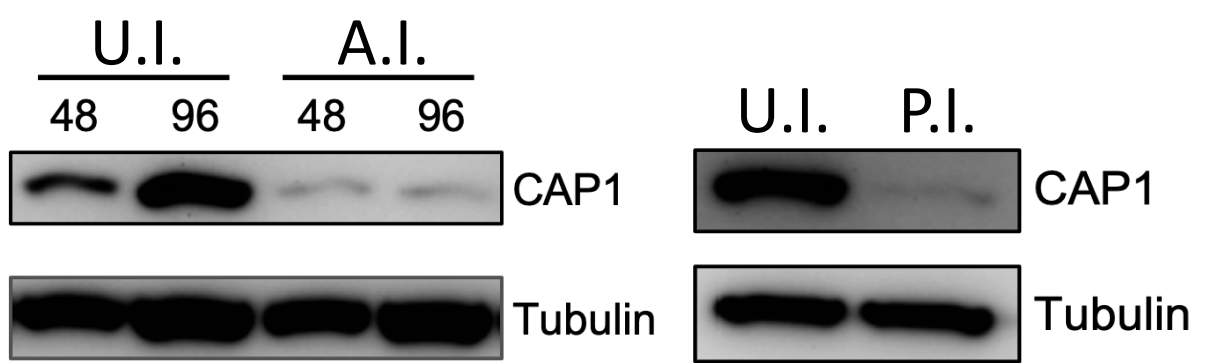

C 506

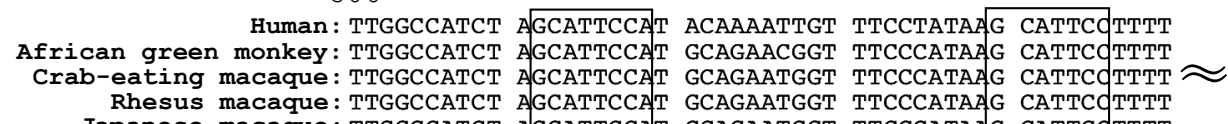

768 CTGGCATTCC Rhesus macaque: TTGGCCATCT AGCATTCCAT GCAGAATGGT TTCCCATAAG CATTCOTTTT
Japanese macaque: TTGGCCATCT AGCATTCCAT GCAGAATGGT TTCCCATAA CATTCOTTTT

Fig. 2. Reduction in the expression of CAP1 by an infection with SFVmfu in TE671 cells. (A) Total RNAs of uninfected TE671 and TE671/ $\mathrm{SFVmfu}(\mathrm{PI})$ cells were extracted and subjected to transcriptome analyses. The gray and black dots indicate the genes that were predicted to have low and high affinities with SFVmfu-miR-S7-5p, respectively (see also Table S2.). (B) Immunoblot analyses to detect CAP1. TE671 cells that were either acutely (left panel) or persistently (right panel) infected with SFVmfu were subjected to immunoblot analyses. The numbers on the lanes in the left panel indicate hours post-infection. Abbreviations: U.I.: uninfected TE671 cells; A.I.: TE671 cells acutely infected with SFVmfu; P.I.: TE671/SFVmfu(PI) cells. (C) Three predicted recognition sites for SFVmfu-miR-S7-5p present in CAP1 3'UTR from five different primate species. The numbers above the alignment indicate the locations of CAP1 3'UTR in the human genome.

\section{Titration of SFVmfu}

To titrate stock viruses of SFVmfu, the LacZ assay was performed using $\mathrm{MD}(\mathrm{SFVmfu}-\mathrm{lac} Z)$ cells as indicator cells. Briefly, $2 \times 10^{4}$ of $\mathrm{MD}(\mathrm{SFVmfu}-\mathrm{lacZ})$ cells were plated in each well of a $96-$ well plate (Thermo Fisher Scientific) $1 \mathrm{~d}$ before the inoculation. After $1 \mathrm{~d}$, stock viruses were serially diluted and immediately inoculated into these cells under the presence of polybrene $\left(8 \mu \mathrm{g} \mathrm{mL} \mathrm{m}^{-1}\right)$, and the inoculated cells were incubated for viral absorption for $4 \mathrm{~h}$ at $37^{\circ} \mathrm{C}$ in a humidified atmosphere of $5 \% \mathrm{CO}_{2}$ in air. After the inoculation, inocula were replaced with $0.2 \mathrm{~mL}$ of fresh medium, and further incubated for an additional $2 \mathrm{~d}$. Cells were then fixed with $1 \%$ glutaraldehyde and stained with X-Gal (5bromo-4-chloro-3-indolyl- $\beta$-D-galactopyranoside), and lacZpositive foci were counted for titration as described previously (Sakaguchi et al., 2008; Hashimoto-Gotoh et al., 2015).

\section{Establishment of TE671 cells persistently infected with SFVmfu}

TE671 cells persistently infected with the SFVmfu strain WK1 (clone JM356) were established as described previously (Miyazawa et al., 1995). Briefly, SFVmfu was inoculated into TE671 cells at a multiplicity of infection (MOI) of 0.172. Cells were cultured and passaged several times until severe cytopathic effects (syncytial formation and cell death) were observed. After the majority of cells had detached from the culture plate, surviving cells were co-cultured with uninfected TE671 cells. The cocultured cells continued to grow, showing mild cytopathic effects. After several passages, cells were able to be passaged regularly (2-4-fold dilution per 4-5 d). We designated these cells as TE671/ SFVmfu(PI) cells.

\section{Construction of plasmids}

Genomic DNAs were extracted from uninfected TE671 and TE671/SFVmfu(PI) cells using the QIAamp DNA Blood Mini kit 
(QIAGEN). Genomic DNAs were used as templates to construct miRNA expression plasmids. All primers used in the present study are listed in Table S1. PCR was performed using KOD-Plus-Neo (Toyobo) according to the manufacturer's instructions. In PCR, we used $200-\mu \mathrm{L}$ thin-walled tubes and a C1000 thermal cycler (BioRad Laboratories). Regarding hsa-mir-1, the amplified fragment was cloned into pcDNA3.1(+) digested with $X b a \mathrm{I} / X h o \mathrm{I}$ to become pcDNA3.1(+)/hsa-mir-1 (Fig. 3A [top]). The amplified fragment for SFVmfu-mir-S6-7 was cloned into pUC18 digested with HindIII/BamHI to become pUC18/SFVmfu-mir-S6-7 (Fig. 3A [center]).
A series of firefly luciferase reporter plasmids was constructed with inserts at the 3 '-end of firefly luciferase. Briefly, the human cytomegalovirus (hCMV) enhancer/promoter was cloned into the NheI/HindIII sites of pGL3-basic (Promega) to become pGL3hCMV (Fig. 3A [bottom]). The complementary sequence of each mature miRNA (hsa-miR-1 or SFVmfu-miR-S7-5p) was prepared by annealing and extending the synthetic oligo and cloned into the $X b a I$ site of the pGL3-hCMV using NEBuilder HiFi DNA Assembly (New England Biolab) to become pGL3-hCMV/hsa-miR-1 or pGL3/hCMV/SFVmfu-miR-S7-5p (Fig. 3A [bottom]). Similarly, the 3'UTR of human CAP1 was cloned into the XbaI site of pGL3-

\section{A}

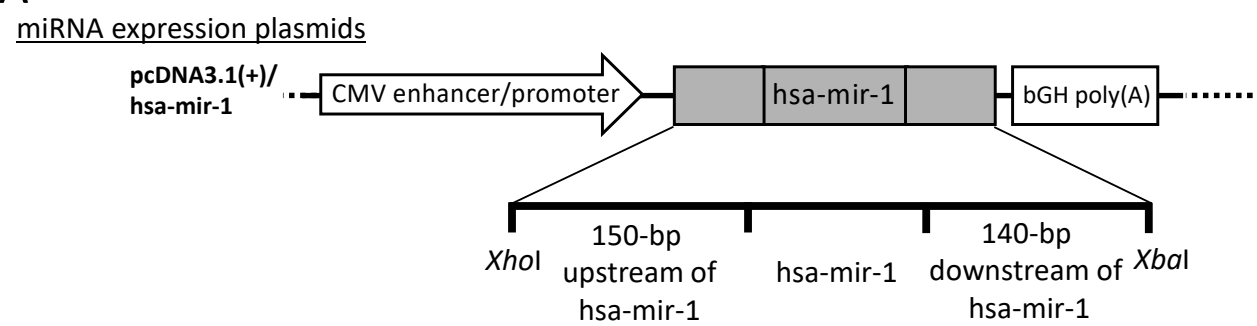

pUC18/

SFVmfu-mir-S6-7

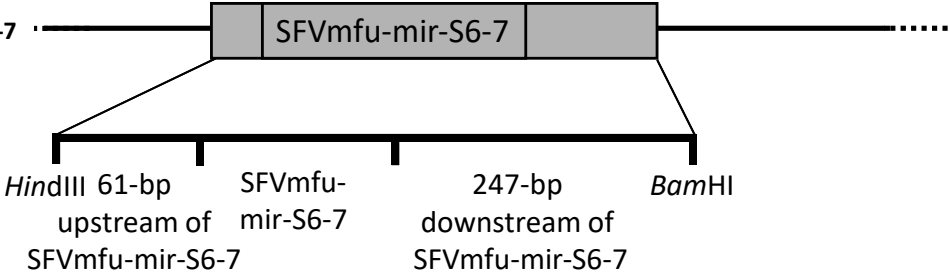

$\underline{\text { Reporter plasmids }}$

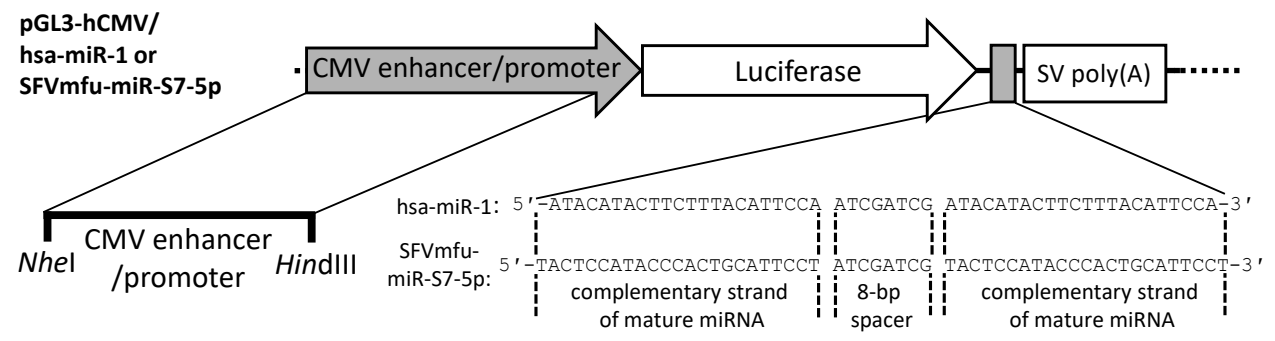

B
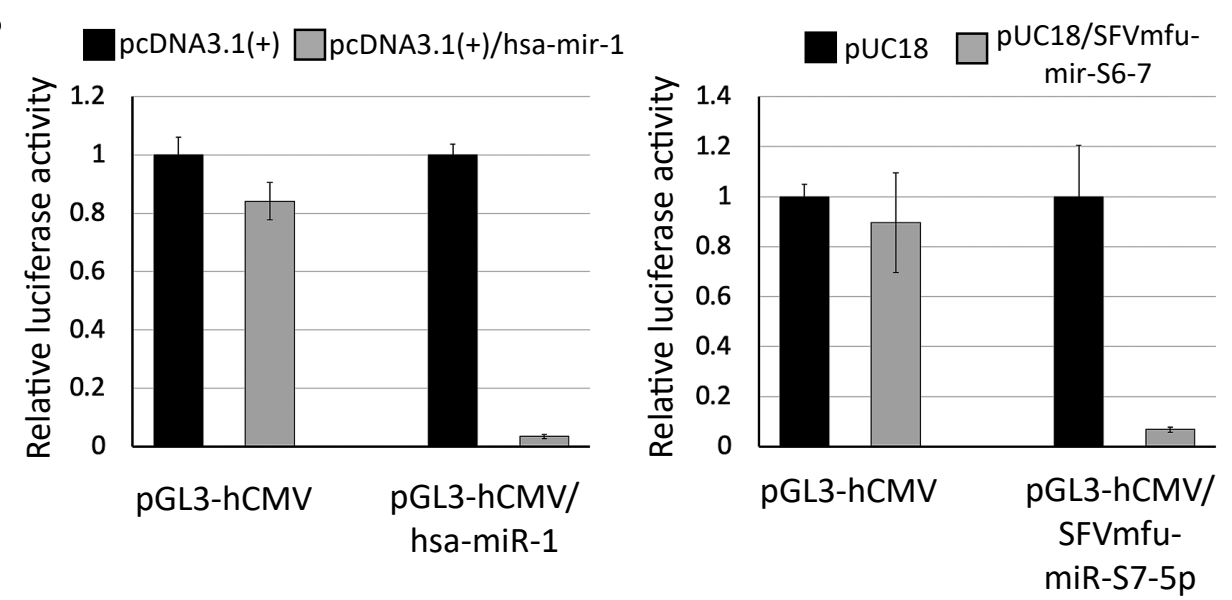

Fig. 3. RISC activity assays for miRNA expression plasmids. (A) Expression plasmids constructed for hsa-mir-1 (top) and SFVmfu-mir-S6-7 (center), and reporter plasmids constructed for hsa-miR-1 and SFVmfu-miR-S7-5p (bottom). The backbone plasmid (pUC18) for SFVmfu-mirR6-7 does not contain mammalian promoters; however, the pre-miRNA sequence contains the internal promoter elements (A/B-box sequences). The reporter plasmids contain complementary sequences of mature miRNAs between the firefly luciferase gene and poly(A) signal of the pGL3 vector inserted with the hCMV enhancer/promoter. Sections cloned into the backbone plasmids are colored in grey. (B) RISC activity assays using the expression plasmids of hsa-miR-1 (left panel) and SFVmfu-miR-S7-5p (right panel). Abbreviations: bGH: bovine growth hormone; SV: simian virus 40 . 


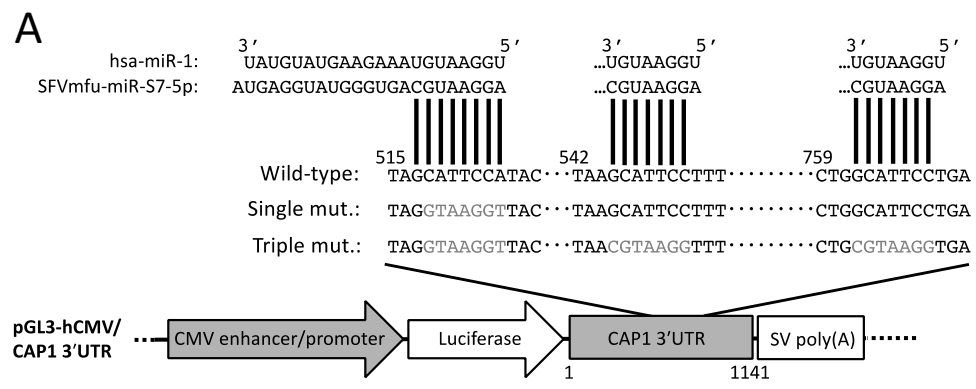

B

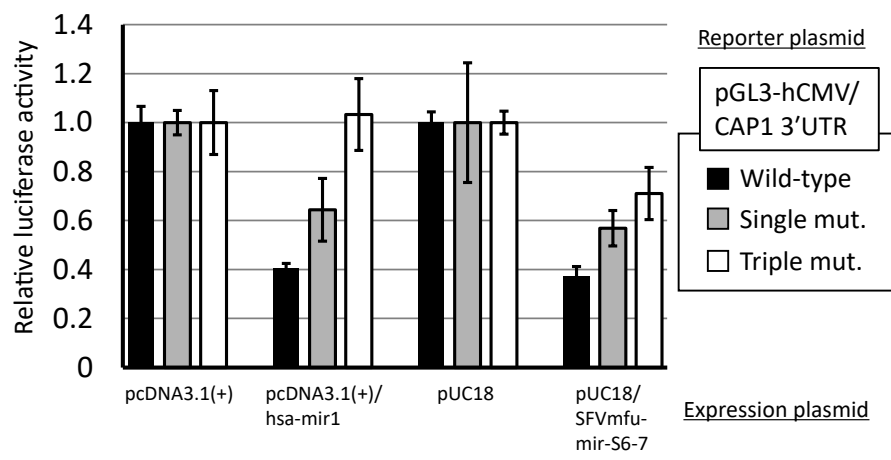

C

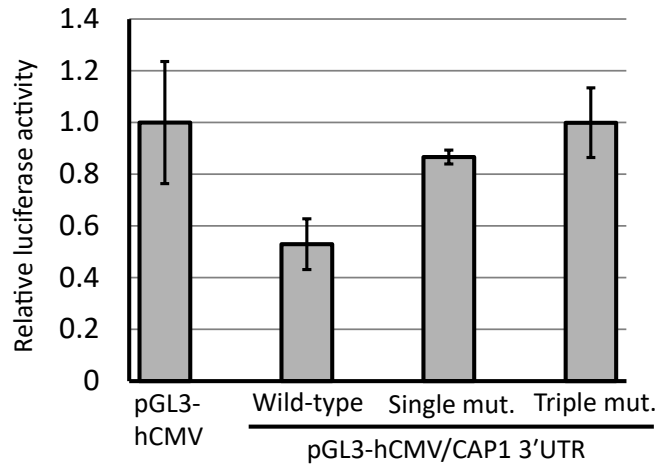

Fig. 4. Suppression of CAP1 expression by SFVmfu-miR-S7-5p via recognition sites. (A) Reporter plasmids containing the 3'UTR of the CAP1 gene. Nucleotides shown in grey characters indicate mutated miRNA recognition sites. The numbers above the alignment indicate the locations of CAP1 3'UTR in the human genome. (B) A luciferase reporter assay was performed by the co-transfection of miRNA expression plasmids with reporter plasmids. (C) A luciferase reporter assay was performed using TE671/SFVmfu(PI) cells without miRNA expression plasmids.

hCMV to become pGL3-hCMV/CAP1 3'UTR (Wild-type) (Fig. 4A). Using pGL3-hCMV/CAP1 3'UTR (Wild-type) as a template, site-directed mutagenesis was performed at the hsa-miR-1/ SFVmfu-miR-S7-5p recognition sites within CAP1 3'UTR to become pGL3-hCMV/CAP1 3'UTR (Single mutant [mut.]) and pGL3-hCMV/CAP1 3'UTR (Triple mut.) (Fig. 4A). Recognition sites were predicted using the online database miRDB version 6.0 (http://www.mirdb.org/) (Wong and Wang, 2015; Liu and Wang, 2019).

\section{Next-generation sequencing analysis}

Small RNA sequencing and transcriptome analyses were performed on TE671 and TE671/SFVmfu(PI) cells. Total RNAs were extracted from cells using RNAzol (Promega), followed by a DNase I (Roche) treatment without a heat inactivation step, and reextracted using RNAzol. Re-extracted RNAs were further processed by a commercial next-generation sequencing company (Novogene).

Regarding small RNA sequencing, sequencing libraries were constructed from total RNAs using the NEBNext Multiplex Small RNA Library Prep Set for Illumina (New England Biolab), which generates strand-specific small RNA libraries. Size selection of the cDNA libraries was performed using native polyacrylamide gel (12\%)-electrophoresis to isolate a fraction containing miRNAderived cDNAs. Single-end 50-bp sequencing was then performed on an Illumina Hiseq 2500 platform. Sequencing adapter sequences were trimmed from the pre-processed reads using Trimmomatic (ver. 0.38) followed by Fastp (ver. 0.19.4) (Bolger et al., 2014; Chen et al., 2018). Reads were then mapped to the viral genome of the SFVmfu clone JM356 (Yoshikawa et al., 2014) using Bowtie2 (ver. 2.3.4.3) (Langmead and Salzberg, 2012) and converted to the bam file using Samtools (ver. 1.9) (Li et al., 2009). Bam files were converted to bed files, and the coverage depth was calculated using Bedtools (ver. 2.28.0) (Quinlan and Hall, 2010). The secondary structures of miRNA were predicted using RNAfold with default parameters (Gruber et al., 2008; Lorenz et al., 2011). Coverage depth was visualized using Microsoft Excel (ver. 16.28).

In transcriptome analyses, sequencing libraries were constructed using the NEBNext Ultra RNA Library Prep Kit for Illumina (New England Biolab), which generates non-strand-specific cDNA libraries. Paired-end 150-bp sequencing was performed on an Illumina Hiseq 2500 platform. Reads were mapped to the human genome (GRCh38.p12) using Hisat2 (ver. 2.1.0) (Kim et al., 2015) and converted to a bam file using Samtools (ver. 1.9) (Li et al., 2009). The mapped reads were assembled using Cufflinks (ver. 2.1.1), and expression differences between uninfected TE671 and TE671/ SFVmfu(PI) cells were calculated using Cuffmerge (ver. 2.1.1) and Cuffdiff (ver. 2.1.1) (Trapnell et al., 2010; 2012). The potential targets of SFVmfu-miR-S7-5p were predicted using the online data- 
base miRDB version 6 (Wong and Wang, 2015; Liu and Wang, 2019). Calculated expression levels were visualized using Microsoft Excel (ver. 16.28).

Multiple sequencing alignment analyses of CAP1 3'UTRs and SFV LTRs from various species

The genomic DNA of a Japanese macaque was extracted from blood using the QIAamp DNA Blood Mini kit (QIAGEN). Extracted DNA was used as a template to amplify the 3'UTR of CAP1 by PCR using the primers listed in Table S1. The amplified product was cloned into the HindIII/EcoRI sites of pcDNA3.1(+). The reverse primer used for PCR was applied for sequencing. A sequencing analysis was performed by a commercial DNA sequencing company (Fasmac). The 3'UTR sequences of CAP1 from humans (Homo sapiens), African green monkeys (AGM), crab-eating macaques (M. fascicularis), and rhesus macaques ( $M$. mulatta) were retrieved from ENSEMBL release 97 (human: GRCh38.p12, vervet AGM: ChlSab1.1, crab-eating macaque: Macaca_fascicularis_5.0, rhesus macaque: Mmul_8.0.1) (Accessed in July 2019).

The LTR sequences of the following SFVs were retrieved from the National Center of Biotechnology Information: SFVcae_LK3 (NC 010820), SFVmcy FV34 (KF026286.1), SFVmmu DPZ99524 (MG051205.1) and SFVmfu_WK1.pJM356 (NC_039026.1), which are derived from AGM, Taiwanese macaques (M. cyclopis), rhesus macaques, and Japanese macaques, respectively.

Multiple sequence alignment analyses of CAP1 3'UTRs and SFV LTRs were performed using the MAFFT program (ver. 7.407) with the L-INS-i method (Katoh and Standley, 2013). The aligned sequences were visualized using Seaview (ver. 4.7) (Gouy et al., 2010).

\section{Immunoblot analyses}

Whole cell lysates of TE671 cells were obtained with RIPA buffer $(50 \mathrm{mM}$ Tris-Cl [pH 7.4], $150 \mathrm{mM} \mathrm{NaCl}, 5 \mathrm{mM}$ EDTA, $1 \%$ Nonidet P-40, $1 \%$ sodium deoxycholate, $0.1 \%$ sodium dodecyl sulfate, $1 \%$ aprotinin, $50 \mathrm{mM} \mathrm{NaF}$, and $\left.0.1 \mathrm{mM} \mathrm{Na}_{3} \mathrm{VO}_{4}\right) 48$ or $96 \mathrm{~h}$ after the mock or SFVmfu infection (at an MOI of 0.34). Similarly, whole cell lysates of uninfected TE671 and TE671/SFVmfu(PI) cells were obtained with RIPA buffer. Lysates were mixed with Laemmli sample buffer supplemented with 2-mercaptoethanol and boiled at $95^{\circ} \mathrm{C}$ for $5 \mathrm{~min}$. Each boiled sample was electrophoresed on a sodium dodecyl sulfate-polyacrylamide gel and electrically blotted onto a polyvinylidene difluoride membrane. Each blotted membrane was blocked in 5\% milk/Tris-buffered saline with Tween 20 and incubated with an anti-CAP1 antibody (Abcam [catalog number: EPR8339\{B\}]) as the primary antibody or a goat anti-rabbit IgG-horseradish peroxidase antibody (Thermo Fisher Scientific [catalog number: 31466]) as the secondary antibody. Antibody reactions were detected and processed with the SuperSignal West Femto system (Thermo Fisher Scientific) using a Luminescent Image Analyzer LAS4000 mini (Fujifilm).

\section{RISC activity assay}

The RISC activity assay was conducted to verify that miRNA expression plasmids produce the intended miRNAs. HEK293T cells in collagen-coated 24 -well plates (Iwaki) were co-transfected with $40 \mathrm{ng}$ of each firefly luciferase reporter plasmid, $4 \mathrm{ng}$ of $\mathrm{pRL}$ TK (Renilla luciferase reporter plasmid) (Promega), and $456 \mathrm{ng}$ of each expression plasmid, as indicated in Fig. 3B, using Lipofectamine 2000 (Thermo Fisher Scientific) according to the manufacturer's instructions. Cells were harvested $24 \mathrm{~h}$ after transfection and subjected to the luciferase assay with the Dual-Glo Luciferase Assay System (Promega) using Lumat LB9507 (Berthold). The significance of differences was assessed using the Student's $t$-test.

\section{Host target 3'UTR luciferase assays}

HEK293T cells in collagen-coated 24-well plates (Iwaki) were co-transfected with $5 \mathrm{ng}$ of pGL3-hCMV/CAP1 3'UTR (either
Wild-type, Single mut., or Triple mut.), $5 \mathrm{ng}$ of pRL-TK (Promega), and $790 \mathrm{ng}$ of either empty plasmid (pUC18 or pcDNA3.1[+]) or each miRNA expression plasmid, as indicated in Fig. 4B, in triplicate using Lipofectamine 2000 (Thermo Fisher Scientific) according to the manufacturer's instructions. Cells were harvested $24 \mathrm{~h}$ after transfection and subjected to the luciferase assay with the Dual-Glo Luciferase Assay System (Promega) using Lumat LB9507 (Berthold). The significance of differences was assessed using the Student's $t$-test.

Uninfected TE671 and TE671/SFVmfu(PI) cells in collagencoated 24-well plates (Iwaki) were co-transfected with $80 \mathrm{ng}$ of pGL3-hCMV/CAP1 3'UTR (either Wild-type, Single mut., or Triple mut.), or pGL3-hCMV, $80 \mathrm{ng}$ of pRL-TK, and $640 \mathrm{ng}$ of the empty plasmid (pcDNA3.1[+]) in triplicate using Lipofectamine 2000 (Thermo Fisher Scientific) according to the manufacturer's instructions. Cells were harvested $24 \mathrm{~h}$ after transfection and assayed for luciferase activity with the Dual-Glo Luciferase Assay System (Promega) using Lumat LB9507 (Berthold). The significance of differences was assessed using the Student's $t$-test.

\section{Accession numbers}

Illumina Hiseq sequencing data and nucleotide sequences identified in the present study were deposited to the database of the DNA Data Bank of Japan with the following accession numbers: DRA008912, DRA008923, and LC498639.

\section{Results}

TE671 cells persistently infected with SFVmfu express viral miRNAs

Small RNA sequencing analyses were performed using total RNAs extracted from both TE671 and TE671/ SFVmfu(PI) cells. Almost all short reads (99.99\%) obtained from uninfected TE671 cells were not mapped to the viral genome of SFVmfu, whereas approximately one-third of short reads $(32.02 \%)$ obtained from TE671/SFVmfu(PI) cells were mapped to the viral genome of SFVmfu (Fig. 1A). As reported previously in SFVcae (Kincaid et al., 2014), seven pre-miRNAs (S1-S7) and 14 mature-miRNAs were identified in SFVmfu, all of which were derived from the $\mathrm{U} 3$ region of LTR (Fig. 1B and S1, and Table 1). Moreover, the pre-miRNAs identified contained A/B-box sequences, which are the essential promoter elements for initiating transcription driven by RNA polymerase III (Fig. 1C and S1) (Kassavetis and Geiduschek, 2006). These promoter elements were also identified in the pre-miRNAs of SFVcae (Kincaid et al., 2014). Among the 14 mature miRNAs identified, SFVmfu-miR-S7-5p, which showed the highest expression level, was derived from a dumbbell-shaped premiRNA, SFVmfu-mir-S6-7 (Fig. 1C and Table 1). The seed sequence of SFVmfu-miR-S7-5p ("GGAAUG") was different from that of SFVcae-miR-S7-5p ("AGAAGG") (Fig. 1D). Notably, the miR-1 miRNA precursor family of the host had the same seed sequence as SFVmfu-miR-S7-5p (Fig. 1D). These results indicate that SFVmfu-miR-S7-5p shares the same target genes as human miR-1 (hsa-miR-1).

\section{CAP1 is down-regulated by SFVmfu infection in TE671 cells}

To clarify the function of SFVmfu-miR-S7-5p, a transcriptome analysis was performed in both TE671 and TE671/SFVmfu(PI) cells (Fig. 2A). Among the genes predicted to be recognized by SFVmfu-miR-S7-5p (highlighted with black dots in Fig. 2A), the expression level of CAP1 
Table 1. List of mature miRNA sequences identified in this study

\begin{tabular}{|c|c|c|c|c|c|c|}
\hline Name & Sequence $\left(5^{\prime}-3^{\prime}\right)$ & $\begin{array}{c}\text { Length } \\
\text { (nt) }\end{array}$ & $\begin{array}{c}\text { Shape of } \\
\text { pre-miRNA }\end{array}$ & $\begin{array}{c}\text { Start } \\
\text { position }\end{array}$ & $\begin{array}{c}\text { End } \\
\text { position }\end{array}$ & $\begin{array}{l}\text { Number } \\
\text { of reads }\end{array}$ \\
\hline SFVmfu-miR-S1-5p & AGGAAGCAUUUGGUAAAUUCUAC & 23 & \multirow{2}{*}{ Dumbbell shape with S2 } & 10,476 & 10,498 & 121 \\
\hline SFVmfu-miR-S1-3p & UCAUUUACUACCGUGCUUCCGA & 22 & & 10,512 & 10,533 & 135,125 \\
\hline SFVmfu-miR-S2-5p & UGGAGAACUUAGGGACGAGGCU & 22 & \multirow{2}{*}{ Dumbbell shape with S1 } & 10,534 & 10,555 & 655,144 \\
\hline SFVmfu-miR-S2-3p & UCCUCAUCUCGAGUGUCUCCCUUU & 24 & & 10,571 & 10,594 & 20,786 \\
\hline SFVmfu-miR-S3-5p & AAGGGAGGGAGUGGAACGUCCUG & 23 & \multirow{2}{*}{ Single hairpin } & 10,658 & 10,680 & 35,207 \\
\hline SFVmfu-miR-S3-3p & UGACGCUCUCCCAUCCCUCCUUUUC & 25 & & 10,695 & 10,719 & 67,292 \\
\hline SFVmfu-miR-S4-5p & UCAAGAACCCAGGGAGCAAUGUUG & 24 & \multirow{2}{*}{ Single hairpin } & 10,842 & 10,865 & 250,036 \\
\hline SFVmfu-miR-S4-3p & AUAGUGCAUCCUGGUCGUUCUUUA & 24 & & 10,881 & 10,904 & 55,352 \\
\hline SFVmfu-miR-S5-5p & ACAAAGGGAAAUAGCUAAUGUGCA & 24 & \multirow{2}{*}{ Single hairpin } & 10,961 & 10,984 & 44 \\
\hline SFVmfu-miR-S5-3p & UACUUAGCCGUUCUCCUUUGAUA & 23 & & 10,999 & 11,021 & 91,370 \\
\hline SFVmfu-miR-S6-5p & AGGUGAAUGGCUCACAGUGAACGA & 24 & \multirow{2}{*}{ Dumbbell shape with S7 } & 11,120 & 11,143 & 57,461 \\
\hline SFVmfu-miR-S6-3p & UACGCUGCGUUGCCACCACCU & 21 & & 11,159 & 11,179 & 620,914 \\
\hline SFVmfu-miR-S7-5p & AGGAAUGCAGUGGGUAUGGAGUA & 23 & \multirow{2}{*}{ Dumbbell shape with S6 } & 11,182 & 11,204 & $1,014,472$ \\
\hline SFVmfu-miR-S7-3p & UUCAUACUAACUACAUUCUUUU & 22 & & 11,222 & 11,243 & 434 \\
\hline
\end{tabular}

was relatively high in uninfected TE671 cells, but low in TE671/SFVmfu(PI) cells (app. 75\% reduction of FPKM). The immunoblot analysis also indicated that the expression of CAP1 was severely down-regulated at the protein level by both acute and persistent infections with SFVmfu (Fig. 2B). The 3'UTR of CAP1 contained three potential SFVmfu-miR-S7-5p recognition sites, which were conserved across several primate species, including the Japanese macaque and humans (Fig. 2C).

SFVmfu-miR-S7-5p down-regulates a reporter gene with its complementary sequence in $3^{\prime} U T R$

The RISC activity assay was performed to investigate whether the expression plasmids constructed for SFVmfumir-S7-5p and hsa-mir-1 (pUC18/SFVmfu-mir-S6-7 and pcDNA3.1[+]/hsa-mir-1, respectively) produce targetspecific miRNAs. A small part of the U3 region of LTR in SFVmfu was used to construct pUC18/SFVmfu-mir-S6-7 (containing the sequence of SFVmfu-miR-S7-5p) in order to avoid overlaps with other viral miRNAs (Fig. 3A [center]). Reporter plasmids were also constructed, which contained the complementary sequences of hsa-miR-1 and SFVmfumiR-S7-5p downstream of the firefly luciferase gene (Fig. $3 \mathrm{~A}$ [bottom]). The results obtained showed that both miRNA expression plasmids down-regulated the expression of the reporter plasmids with complementary sequences (Fig. 3B), indicating that both expression plasmids produced functional miRNAs (hsa-miR-1 and SFVmfu-miR-S7-5p, respectively), which silenced the mRNAs containing complementary sequences in 3'UTR.

\section{SFVmfu-miR-S7-5p suppresses CAP1 expression via recognition sites in $3^{\prime} U T R$}

To investigate whether SFVmfu-miR-S7-5p recognizes and down-regulates the expression of a gene bearing the 3'UTR of CAP1, we constructed a series of reporter plasmids containing either the wild-type or modified 3'UTR of CAP1 downstream of the firefly luciferase gene (Fig. 4A). hsa-miR-1 and SFVmfu-miR-S7-5p both repressed the expression of luciferase when the reporter plasmids contained the wild-type 3'UTR of CAP1 (Fig. 4B). Luciferase expression levels were restored when the predicted recogni- tion sites were modified (Fig. 4B), suggesting that hsamiR-1 and SFVmfu-miR-S7-5p both repress CAP1 expression via the recognition sites.

An additional reporter assay was performed on TE671/ SFVmfu(PI) cells without miRNA expression plasmids. In TE671/SFVmfu(PI) cells, the level of luciferase expression was suppressed when the reporter plasmid contained the wild-type 3'UTR of CAP1 and was restored when the recognition sites were disrupted (Fig. 4C). Based on these results, we concluded that TE671/SFVmfu(PI) cells express SFVmfu-miR-S7-5p, which is capable of down-regulating CAP1 expression via recognition sites.

\section{Discussion}

In the present study, we showed that persistent infection with SFVmfu in TE671 cells increased the expression levels of viral miRNAs encoded in the U3 region of SFVmfu LTR, as previously reported in SFVcae (Kincaid et al., 2014) (Fig. 1). Among the miRNAs identified, we focused our analyses on SFVmfu-miR-S7-5p, which had the highest read count among the 14 viral miRNAs identified (Fig. 1B). A multiple sequence alignment analysis of SFVs from Macaca species indicated that the U3 region of SFVmfu was distinct, but similar to those of SFVs from other macaques (rhesus and Taiwanese macaques [SFVmmu strain DPZ9524 and SFVmcy strain FV34, respectively]) (Fig. S2). Notably, the seed sequence ("GGAAUG") of miRS7-5p of SFVmfu was the same as those of SFVmmu and SFVmcy, suggesting that they also have the ability to express miRNA harboring the same seed sequence as SFVmfu-miR-S7-5p. On the other hand, the seed sequence ("AGAAGG") of SFVcae-miR-S7-5p was markedly different from that of SFVmfu-miR-S7-5p and only moderately expressed (Kincaid et al., 2014). Instead, cells infected with SFVcae expressed SFVcae-miR-S6-3p at a high level, which has the same seed sequence ("AACAGUC") as hsamiR-132 (Kincaid et al., 2014). Moreover, SFVcae-miRS6-3p and hsa-miR-132 both suppressed the expression of EP300 (Kincaid et al., 2014), which is the transcriptional coactivator of the type-1 interferon response (Lagos et al., 2010). However, cells infected with SFVmfu expressed 
SFVmfu-miR-S6-3p with a different seed sequence ("CGCUGCG") only at a moderate level (Fig. 1B and S2). Our transcriptome analyses indicated that the expression of EP300 was not significantly changed by the SFVmfu infection (data not shown). These results suggest that the target genes regulated by miRNAs differ between SFVmfu and SFVcae.

The expression of CAP1 was severely down-regulated by the SFVmfu infection in TE671 cells (Fig. 2). In the luciferase reporter assay using miRNA expression plasmids, the luciferase activity of the reporter plasmid containing CAP1 3'UTR was not fully recovered by the disruption of all three predicted miRNA recognition sites (Fig. 4B). On the other hand, when the reporter plasmids were transfected into TE671/SFVmfu(PI) cells, luciferase activity was fully recovered by the disruption of the three recognition sites (Fig. 4C). One possible explanation for this discrepancy is that the use of the plasmid (pUC18) without mammalian promoters may cause the non-specific suppression of the firefly luciferase protein produced from pGL3-based reporter plasmids. This incomplete recovery associated with using a plasmid without mammalian promoters in the luciferase reporter assay was also observed in a previous study (Kincaid et al., 2014). Nonetheless, the complete recovery of relative luciferase activity, in the reporter assay using TE671/SFVmfu(PI) cells, by the disruption of the three recognition sites indicates that SFVmfu-miR-S7-5p downregulated CAP1 through the predicted recognition sites in cells.

Physiologically, CAP1 is involved in actin cytoskeleton remodeling in mammalian cells, and the knockdown of CAP1 results in impaired cell migration with decreased actin depolymerization (Bertling et al., 2004). Actin depolymerization plays a crucial role in the replication of retroviruses, such as human immunodeficiency virus 1 (Yoder et al., 2008; Gladnikoff et al., 2009; Santos et al., 2012; Wen et al., 2014). However, it currently remains unclear whether the down-regulation of CAP1 by SFVmfu-miR-S7-5p benefits the replication strategy of SFVmfu.

One possible hypothesis for the significance of SFV miRNAs is that viral miRNAs contribute to the autoinhibitory regulation of SFV gene expression in infected cells. The deletion of the 5'-portion of the U3 region (a possible miRNA cluster) in a chimpanzee-derived SFV was shown to increase viral replication in fibroblasts (Schmidt et al., 1997). SFV may utilize miRNAs to optimize long-term coexistence with the host, as reported for other viruses, such as herpes simplex virus 1 and polyomavirus (Umbach et al., 2008; Broekema and Imperiale, 2013).

Another hypothesis for the significance of SFV miRNAs is that the miRNAs of SFVs suppress the expression of genes that are involved in the development of cancers. Among the family Retroviridae, gammaretroviruses preferentially integrate into the vicinity of the TSSs of the host genes (Desfarges and Ciuffi, 2010). This integration potentially leads to tumorigenesis when integration occurs in the promoter/enhancer regions of proto-oncogenes. Similar to gammaretroviruses, FVs are also more likely to integrate into the vicinity of TSSs (Trobridge et al., 2006). However, the development of cancer by SFV infection has not yet been reported. Thus, SFVs may utilize as-yet-unknown mechanisms to avoid the development of cancer in host primates. SFVmfu-miR-S7-5p may counter the development of tumors by repressing the expression of CAP1 in infected cells. It is interesting to note that the expression level of hsamiR-1, which shares a seed sequence with SFVmfu-miR$\mathrm{S} 7-5 \mathrm{p}$, was down-regulated in various types of cancers (Liu et al., 2015; Han et al., 2017; Korde et al., 2017). The upregulation of CAP1 has been observed in several solid tumors, such as ovarian cancer, breast cancer (Xie et al., 2014; 2018; Hua et al., 2015; Hasan and Zhou, 2019), and rhabdomyosarcoma from which the TE671 cell line is derived (Rao et al., 2010). Rhabdomyosarcoma is also known to have a reduced expression level of hsa-miR-1, which suppresses CAP1 expression (Vinther et al., 2006; Rao et al., 2010). Interestingly, SFVcae-miR-S6-3p shares a seed sequence with hsa-miR-132, which is also downregulated in several types of tumors (Formosa et al., 2012; Wang et al., 2014; Zhang et al., 2016). SFVs may utilize distinct miRNAs to avoid tumorigenesis by suppressing various onco-related genes.

\section{Acknowledgements}

We would like to thank the Japan Society for the Promotion of Science (JSPS) for providing financial support for this project (KAKENHI Grant Numbers 16J10964, 17H03924, and 17H01048). A. H.-G. received financial support from the JSPS Research Fellowship for Young Scientists.

\section{References}

Abdelfattah, A.M., Park, C., and Choi, M.Y. (2014) Update on noncanonical microRNAs. Biomol Concepts 5: 275-287.

Bartel, D.P. (2009) MicroRNAs: target recognition and regulatory functions. Cell 136: 215-233.

Bertling, E., Hotulainen, P., Mattila, P.K., Matilainen, T., Salminen, M., and Lappalainen, P. (2004) Cyclase-associated protein 1 (CAP1) promotes cofilin-induced actin dynamics in mammalian nonmuscle cells. Mol Biol Cell 15: 2324-2334.

Bolger, A.M., Lohse, M., and Usadel, B. (2014) Trimmomatic; a flexible trimmer for illumine sequence data. Bioinformatics 30: 2114-2120.

Broekema, N.M., and Imperiale, M.J. (2013) miRNA regulation of BK polyoma-virus replication during early infection. Proc Natl Acad Sci $U S A$ 110: 8200-8205.

Cao, W., Heit, A., Hotz-Wagenblatt, A., and Löchelt, M. (2018) Functional characterization of the bovine foamy virus's miRNA expression cassette and its dumbbell-shaped pri-miRNA. Virus Genes 54: 550-560.

Chen, S., Zhou, Y., Chen, Y., and Gu, J. (2018) Fastp: an ultra-fast all-inone FASTQ preprocessor. Bioinformatics 34: 884-890.

Chendrimada, T.P., Gregory, R.I., Kumaraswamy, E., Norman, J., Cooch, N., Nishikura, K., and Shiekhattar, R. (2005) TRBP recruits the Dicer complex to Ago2 for microRNA processing and gene silencing. Nature 436: 740-744.

Desfarges, S., and Ciuffi, A. (2010) Retroviral integration site selection. Viruses 2: 111-130.

Eulalio, A., Huntzinger, E., Nishihara, T., Rehwinkel, J., Fauser, M., and Izaurralde, E. (2009) Deadenylation is a widespread effect of miRNA regulation. $R N A$ 15: 21-32.

Formosa, A., Lena, A.M., Markert, E.K., Cortelli, S., Miano, R., Mauriello, A., et al. (2012) DNA methylation silences miR-132 in prostate cancer. Oncogene 32: 127-134.

Galvin, T.A., Ahmed, I.A., Shahabuddin, M., Bryan, T., and Khan, A.S. (2013) Identification of recombination in the envelope gene of simian foamy virus serotype 2 isolated from Macaca cyclopis. $J$ Virol 87: 8792-8997. 
Gladnikoff, M., Shimoni, E., Gov, N.S., and Rousso, I. (2009) Retroviral assembly and budding occur through an actin-driven mechanism. Biophys J 97: 2419-2428.

Gouy, M., Guindon, S., and Gascuel, O. (2010) SeaView version 4: a multiplatform graphical user interface for sequence alignment and phylogenetical tree building. Mol Biol Evol 27: 221-224.

Graham, F.L., Smiley, J., Russell, W.C., and Nairn, R. (1977) Characteristics of a human cell line transformed by DNA from human adenovirus type 5. J Gen Virol 36: 59-74.

Gruber, A.R., Lorenz, R., Bernhart, S.H., Neuböck, R., and Hofacker, I.L. (2008) The Vienna RNA websuite. Nucleic Acids Res 36: W70-4.

Han, C., Shen, J.K., Hornicek, F.J., Kan, Q., and Duan, Z. (2017) Regulation of microRNA-1 (miR-1) expression in human cancer. Biochim Biophys Acta 1860: 227-232.

Hasan, R., and Zhou, G.-L. (2019) The cytoskeletal protein cyclaseassociated protein 1 (CAP1) in breast cancer: context-dependent roles in both the invasiveness and proliferation of cancer cells and underlying cell signals. Int J Mol Sci 20: E2653.

Hashimoto-Gotoh, A., Yoshikawa, R., and Miyazawa, T. (2015) Comparison between $\mathrm{S}+\mathrm{L}$ - assay and LacZ marker rescue assay for detecting replication-competent gammaretroviruses. Biologicals 43: 363-368.

Hua, M., Yan, S., Yan, D., Xi, Q., Liu, R., Yang, S., et al. (2015) CAP1 is overexpressed in human epithelial ovarian cancer and promotes cell proliferation. Int J Mol Sci 35: 941-949.

Kassavetis, G.A., and Geiduschek, E.P. (2006) Transcription factor TFIIIB and transcription by RNA polymerase III. Biochem Soc Trans 34: 1082-1087.

Katoh, K., and Standley, D.M. (2013) MAFFT multiple sequence alignment software version 7: improvements in performance and usability. Mol Biol Evol 30: 772-780.

Khan, A.S., Bodem, J., Buseyne, J.F., Gessain, A., Johnson, W., Kuhn, J.H., et al. (2018) Spumaretroviruses: updated taxonomy and nomenclature. Virology 516: 158-164.

Kim, D., Langmead, B., and Salzberg, S.L. (2015) HISAT: a fast spliced aligner with low memory requirements. Nat Methods 12: 357-360.

Kincaid, R.P., Burke, J.M., and Sullivan, C.S. (2012) RNA virus microRNA that mimics a B-cell oncomiR. Proc Natl Acad Sci U S A 109: 3077-3082.

Kincaid, R.P., Chen, Y., Cox, J.E., Rethwilm, A., and Sullivana, C.S. (2014) Noncanonical microRNA (miRNA) biogenesis gives rise to retroviral mimics of lymphoproliferative and immunosuppressive host miRNAs. mBio 5: e00074.

Klase, Z., Kale, P., Winograd, R., Gupta, M.V., Heydarian, M., Berro, R., et al. (2007) HIV-1 tar element is processed by dicer to yield a viral micro-RNA involved in chromatin remodeling of the viral LTR. BMC Mol Biol 8: 63.

Korde, A., Jin, L., Zhang, J.G., Ramaswamy, A., Hu, B., Kolahian, S., et al. (2017) Lung endothelial microRNA-1 regulates tumor growth and angiogenesis. Am J Respir Crit Care Med 196: 1443-1455.

Lagos, D., Pollara, G., Henderson, S., Gratrix, F., Fabani, M., Milne, R.S.B., et al. (2010) miR-132 regulates antiviral innate immunity through suppression of the p300 transcriptional co-activator. Nat Cell Biol 12: 513-519.

Lambert, C., Rua, R., Gessain, A., and Buseyne, F. (2016). A new sensitive indicator cell line reveals cross-transactivation of the viral LTR by gorilla and chimpanzee simian foamy viruses. Virology 496 : 219-266.

Lander, M.R., and Chattopadhyay, S.K. (1984) A Mus dunni cell line that lacks sequences closely related to endogenous murine leukemia viruses and can be infected by ectropic, amphotropic, xenotropic, and mink cell focus-forming viruses. J Virol 52: 695-698.

Langmead, B., and Salzberg, S. (2012) Fast gapped-read alignment with bowtie2. Nat Methods 9: 357-359.

Li, H., Handsaker, B., Wysoker, A., Fennel, T., Ruan, J., Homer, N., et al. (2009) The sequence alignment/map format and SAMtools. Bioinformatics 25: 2078-2079.

Liu, T., Hu, K., Zhao, Z., Chen, G., Ou, X., Zhang, H., et al. (2015) MicroRNA-1 down-regulates proliferation and migration of breast cancer stem cells by inhibiting the Wnt/ $\beta$-catenin pathway. Oncotarget 6: 41638-41649.
Liu, W., Worobey, M., Li, Y., Keele, B.F., Bibollet-Ruche, F., Guo, Y., et al. (2008) Molecular ecology and natural history of simian foamy virus infection in wild-living chimpanzees. PLoS Pathog 4: e1000097.

Liu, W., and Wang, X. (2019) Prediction of functional microRNA targets by integrative modeling of microRNA binding and target expression data. Genome Biol 20: 18.

Lorenz, R., Bernhart, S.H., Shiederdissen, C.-H., Tafer, H., Flamm, C., and Stadler, P.F. (2011) ViennaRNA Package 2.0. Algorithms Mol Biol 6: 26.

Miyazawa, T., Itagaki, S., Tomonaga, K., Ikeda, Y., Mori, T., Kawaguchi, Y., et al. (1995) Establishment of carrier state infection of a feline renal cell line with feline syncytial virus. $J$ Vet Med Sci 57: 65-69.

Pinto-Santini, D.M., Stenbak, C.R., and Linial, M.L. (2017) Foamy virus zoonotic infections. Retrovirology 14: 55.

Quinlan, A.R., and Hall, I.M. (2010) BEDtools: a flexible suite of utilities for comparing genomic features. Bioinformatics 26: $841-$ 842.

Rao, P.K., Missiaglia, E., Shields, L., Hyde, G., Yuan, B., Shepherd, C.J., et al. (2010) Distinct roles for miR-1 and miR-133a in the proliferation and differentiation of rhabdomyosarcoma cells. FASEB J 24: 3427-3437.

Sakaguchi, S., Okada, M., Shojima, T., Baba, K., and Miyazawa, T. (2008) Establishment of LacZ marker rescue assay to detect infectious RD114 virus. J Vet Med Sci 70: 785-790.

Santos, S., Obukhov, Y., Nekhai, S., Bukrinsky, M., and Iordanskiy, S. (2012) Virus-producing cells determine the host protein profiles of HIV-1 virion cores. Retrovirology 9: 65.

Schmidt, M., Herchenroder, O., Heeney, J., and Rethwilm, A. (1997) Long terminal repeat U3 length polymorphism of human foamy virus. Virology 230: 167-178.

Stratton, M.R., Darling, J., Pilkington, G.J., Lantos, P.L., Reeves, B.R., and Cooper, C.S. (1989) Characterization of the human cell line TE671. Carcinogenesis 10: 889-905.

Switzer, W.M., Salemi, M., Shanmugam, V., Gao, F., Cong, M., Kuiken, C., et al. (2005) Ancient co-speciation of simian foamy viruses and primates. Nature 434: 376-380.

Thompson, P.J., Macfarlan, T.S., and Lorincz, M.C. (2016) Long terminal repeats: from parasitic elements to building blocks of the transcriptional regulatory repertoire. Mol Cell 62: 766-776.

Trapnell, C., Williams, B., Pertea, G., Mortazavi, A., Kwan, G., Baren, J.V., et al. (2010) Transcript assembly and quantification by RNAseq reveals unannotated transcripts and isoforms switching during cell differentiation. Nat Biotechnol 28: 511-515.

Trapnell, C., Hendrickson, D., Sauvageau, M., Goff, L., Rinn, J.L., and Pachter, L. (2012) Differential analysis of gene regulation at transcript resolution with RNA-seq. Nat Biotechnol 31: 46-53.

Trobridge, G.D., Miller, D.G., Jacobs, M.A., Allen, J.M., Kiem, H.-P., Kaul, R., and Russell, D.W. (2006) Foamy virus vector integration sites in normal human cells. Proc Natl Acad Sci U S A 103: 14981503.

Umbach, J.L., Kramer, M.F., Jurak, I., Karnowski, H.W., Coen, D.M, and Cullen, B.R. (2008) MicroRNAs expressed by herpes simplex virus 1 during latent infection regulate viral mRNAs. Nature 454: 780783.

Vinther, J., Hedegaard, M.M., Gardner, P.P., Andersen, J.S., and Arctander, P. (2006) Identification of miRNA targets with stable isotope labeling by amino acids in cell culture. Nucleic Acids Res 34: e107.

Wang, J., Zu, G., Shen, F., and Kang, Y. (2014) miR-132 targeting cyclin E1 suppresses cell proliferation in osteosarcoma cells. Tumor Biol 35: $4859-4865$.

Wen, X., Ding, L., Wang, J.-J., Qi, M., Hammonds, J., Chu, H., et al. (2014) ROCK1 and LIM kinase modulate retrovirus particle release and cell-cell transmission events. J Virol 88: 6906-6921.

Whisnant, A.W., Kehl, T., Bao, Q., Maternial, M., Kuzmak, J., Löchelt, M., and Cullen, B.R. (2014) Identification of novel, highly expressed retroviral microRNAs in cells infected by bovine foamy virus. $J$ Virol 88: 4679-4686.

Wong, N., and Wang, X. (2015) miRDB: an online resource for microRNA target prediction and functional annotations. Nucleic Acids Res 43: 146-152.

Xie, S., Shen, C., Zhou, S., Xiong, M., Tan, M., Song, X., and Wang, C. (2018) Role of adenylate cyclase-associated protein 1 in cancers. Int J Clin Exp Med 11: 3086-3099. 
Xie, S.S., Tan, M., Lin, H.Y., Xu, L., Shen, C.X., Yuan, Q., et al. (2014) Overexpression of adenylate cyclase-associated protein 1 may predict brain metastasis in non-small cell lung cancer. Oncol Rep 33: 363-371.

Yao, Y., Smith, J.P., Nair, V., and Watson, M. (2014) An avian retrovirus uses canonical expression and processing mechanisms to generate viral microRNA. J Virol 88: 2-9.

Yoder, A., Yu, D., Dong, L., Lyer, S.R., Xu, X., Kelly, J., et al. (2008) HIV envelope-CXCR4 signaling activates cofilin to overcome cortical actin restriction in resting CD4 T cells. Cell 134: 782-792.
Yoshikawa, R., Nakagawa, S., Okamoto, M., and Miyazawa, T. (2014) Construction of an infectious molecular clone of simian foamy virus of Japanese macaque (SFVjm) and phylogenetic analysis of SFVjm isolates. Gene 548: 149-154.

Zhang, X., Tang, W., Li, R., He, R., Gan, T., Luo, Y., et al. (2016) Downregulation of microRNA-132 indicates progression in hepatocellular carcinoma. Exp Ther Med 12: 2095-2101. 